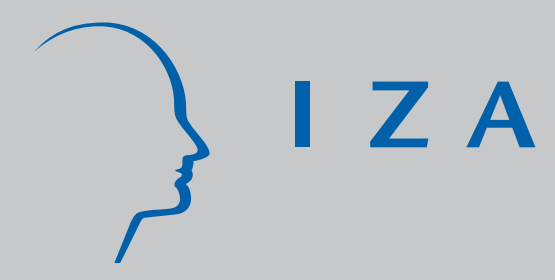

IZA DP No. 1592

Economic Integration, Market Power and Technological Change

Tapio Palokangas

May 2005 


\title{
Economic Integration, Market Power and Technological Change
}

\author{
Tapio Palokangas \\ University of Helsinki, HECER \\ and IZA Bonn
}

\section{Discussion Paper No. 1592 \\ May 2005}

\author{
IZA \\ P.O. Box 7240 \\ 53072 Bonn \\ Germany \\ Phone: +49-228-3894-0 \\ Fax: +49-228-3894-180 \\ Email: iza@iza.org
}

\begin{abstract}
Any opinions expressed here are those of the author(s) and not those of the institute. Research disseminated by IZA may include views on policy, but the institute itself takes no institutional policy positions.

The Institute for the Study of Labor (IZA) in Bonn is a local and virtual international research center and a place of communication between science, politics and business. IZA is an independent nonprofit company supported by Deutsche Post World Net. The center is associated with the University of Bonn and offers a stimulating research environment through its research networks, research support, and visitors and doctoral programs. IZA engages in (i) original and internationally competitive research in all fields of labor economics, (ii) development of policy concepts, and (iii) dissemination of research results and concepts to the interested public.
\end{abstract}

IZA Discussion Papers often represent preliminary work and are circulated to encourage discussion. Citation of such a paper should account for its provisional character. A revised version may be available directly from the author. 


\begin{abstract}

\section{Economic Integration, Market Power and Technological Change*}

We examine a common market which expands by integrating new regions. Capitalists are strategically interdependent through the goods market and they improve their productivity through R\&D. Production and R\&D employ unionized workers. The purpose of integration is to maximize a weighed average of workers' and capitalists' utilities. The main findings are as follows. Integration benefits capitalists more than workers. If labour unions are strong enough, then the common market can expand indefinitely. Otherwise, there is an upper limit for integration. This is the higher, the higher producer market power or the stronger the capitalists' political influence.
\end{abstract}

JEL Classification: F15, J50, O40

Keywords: economic integration, market power, endogenous growth

Corresponding author:

Tapio Palokangas

Department of Economics

P.O. Box 17 (Arkadiankatu 7)

FIN-00014 University of Helsinki

Finland

Email: palokang@valt.helsinki.fi

"The author thanks the participants of the conference on Dynamics, Economic Growth and International Trade (DEGIT), in Iceland in June 2004, and the IZA research seminar in Bonn in September 2004 - in particular Elias Dinopoulos, Theo Eicher, Winfried Koeniger and Uwe Sunde - for useful comments on the earlier drafts of this paper. Financial support from the Yrjö Jahnsson Foundation is gratefully acknowledged. 


\section{Introduction}

In the European Union, strong labour unions and lack of competition have be blamed for slow growth and the difficulty of adopting new members. The reasoning goes as follows. High union wages and high mark-ups in the goods market reduce the demand for output and discourages investment in R\&D. With a lower level of R\&D, there will be less innovations and growth. The expansion of the common market raises opposition, because it reduces unions' and producers' rents. The purpose of this study to examine these assertions.

Using a product-variety model with labour unions and non-competitive firms Peretto (1998) showed that a fall in market power in the labour or goods markets promotes $\mathrm{R} \& \mathrm{D}$ and growth through a higher profit margin. He however assumed that labour is employed only in production, final goods can be directly converted into $\mathrm{R} \& \mathrm{D}$ and labour unions completely ignore the effect of their wages on productivity through R\&D. In this study we, on the contrary, assume that the same homogeneous labour is used both in production and $R \& D$ and unions internalize the effects through $R \& D$.

Dinopoulos and Zhao (2003) examines the interaction of union power and globalization. They as well assume that labour unions ignore the effect of their wages on productivity through $R \& D$ and postulated a union's utility as a geometric average of the wage and employment. They show that macroeconomic effects of globalization depend decisively on the relative weight of the wage in union preferences. In this study, we rather stick to microfoundations and derive union preferences from workers' preferences.

There are already many papers that suggest that expensive labour may speed up economic growth. Cahuc and Michel (1996) (using an OLG model), as well as Agell and Lommerud (1997) (using an extensive game framework) show that a minimum wage may create an incentive for workers to accumulate human capital. Meckl (2004) extends Aghion and Howitt's (1998) Schumpeterian growth model so that production employs skilled and unskilled, but 
R\&D only skilled labour. He shows that higher minimum wages for unskilled labour raise employment of skilled labour and the growth rate.

The same results hold even if the minimum wages are endogenously determined by collective bargaining. Palokangas $(1996,2000)$ introduces wage bargaining into Romer's (1990) product-variety model. He shows that if the elasticity of substitution between skilled and unskilled labour is less than one, then the increase in union bargaining power raises wages for unskilled workers, reduces the demand for skilled workers in production, and thereby lowers wages for skilled workers. This decreases costs in R\&D and promotes growth. Lingens (2003) reconstructs the same effect for Aghion and Howitt's (1998) Schumpeterian growth model. Using Wälde's (1999) Schumpeterian growth model, Palokangas (2005) considers the growth and welfare effects of union power when research firms learn from each other. He shows that the international coordination of labour market policy raises the workers' wages and promotes growth and welfare. In this study, we examine the effect of unions' and producers' market power on economic integration.

Because it is difficult to measure union power, there is still very little empirical evidence on the effects of union power on R\&D and economic growth. ${ }^{1}$ Some papers explain $R \& D$ by the unionization rate, i.e. the ratio of unionized to all workers, ${ }^{2}$ but this is a different issue. ${ }^{3}$ It is not clear either whether

\footnotetext{
${ }^{1}$ Beitnes and Søraas (2003) present some indirect support to a positive dependence of R\&D on union power. They show that the end of de-unionization in South Korea in 1987 increased sharply real wages, $R \& D$ and the accumulation of total factor productivity.

${ }^{2}$ Addison and Wagner (1994) found a positive cross-sectional correlation, but MenezesFilho et.al. (1998) only little correlation in a panel of firms, between R\&D and the unionization rate in the UK. Connolly et.al. (1986), Hirsch (1990; 1992), Bronars et.al. (1994) in the USA, and Betts et.al. (2001) in Canada found a negative cross-sectional correlation between these. Hence, the results have been highly institution-specific.

${ }^{3}$ The unionization rate is not a proper proxy for union power in wage bargaining. In many European countries it tells nothing about union power, because the contract made by the representative union is extended to cover all employers and employees in the industry. In some other countries (e.g. USA, Canada), unions can make agreements only for their members and a unionized worker can be easily replaced by a non-unionized worker. This imposes an additional constraint for the union in wage bargaining, but does not necessarily affect the relative bargaining power of the parties.
} 
unionization increases unemployment. ${ }^{4}$

The basic structure of our model is as follows. We examine a common market with a large number of regions. Households consume the goods of all regions, the workers supply labour but do not save, and the capitalists earn profits and improve their productivity through R\&D. ${ }^{5}$ As a producer, a capitalist takes wages as given and sets its output price. The producers in the common market are strategically interdependent. In each region two parties - a capitalist and workers' union - bargain over wages.

The remainder of this study is organized as follows. Sections 3-5 consider a closed economy with a given number $J$ of regions. R\&D is modelled in section 2 and the goods market in section 3. Section 4 examines the capitalists' behaviour and section 5 wage bargaining. Sections 6 and 7 extend the closed-economy model for a common market with an endogenous number $J$ of regions and consider economic integration that increases $J$.

\section{Technology and R\&D}

Consider a closed economy with a given number $J$ of similar regions. Aggregate consumption $C$ is determined by the CES function

$$
C=J\left(\frac{1}{J} \sum_{j=1}^{J} y_{j}^{1-\gamma}\right)^{1 /(1-\gamma)}
$$

where $y_{j}$ is output in region $j$ and $\gamma \in(0,1)$ the inverse of the constant elasticity of substitution. In each region $j$, there is one producer (hereafter producer $j$ ) with technology

$$
y_{j}=B_{j} n_{j},
$$

\footnotetext{
${ }^{4}$ Belot and van Ours (2001) show that the relationship between union density (= the unionization rate) and the unemployment rate depends on the bargaining structure. When there is decentralized bargaining, an increase in union density causes the unemployment rate to increase. When there is industry level or centralized bargaining, there is no relationship between union density and unemployment.

${ }^{5}$ The households are divided into workers and capitalists, for tractability. It would be difficult to model wage bargaining consistently, if workers owned shares in firms.
} 
where $n_{j}$ is labour input and $B_{j}$ the productivity parameter. We define the average productivity in the economy by the following CES index:

$$
B=\mathcal{B}\left(B_{1}, \ldots, B_{J}\right) \doteq\left(\frac{1}{J} \sum_{j=1}^{J} B_{j}^{1-\gamma}\right)^{1 /(1-\gamma)}
$$

Technology (1)-(3) has the property that with symmetry throughout the regions, $n_{j}=n$ for all $j$, aggregate consumption is in fixed proportion to the size of the economy (= the number of regions) $J$ :

$$
\left.C\right|_{n_{k}=n}=n J\left(\frac{1}{J} \sum_{j=1}^{J} B_{j}^{1-\gamma}\right)^{1 /(1-\gamma)}=n B J .
$$

Hence, there are no scale effects on consumption.

Technological change in region $j$ is characterized by a Poisson process $q_{j}$ as follows. During a short time interval $d \theta$, there is an innovation $d q_{j}=1$ with probability $\Lambda_{j} d \theta$, and no innovation $d q_{j}=0$ with probability $1-\Lambda_{j} d \theta$, where $\Lambda_{j}$ is the arrival rate of innovations in the research process. The arrival rate $\Lambda_{j}$ is in the fixed proportion $\lambda$ to employment in $\mathrm{R} \& \mathrm{D}, l_{j}$,

$$
\Lambda_{j}=\lambda l_{j}
$$

We denote the serial number of technology in region $j$ by $t_{j}$ and variables depending on technology $t_{j}$ by superscript $t_{j}$. The invention of a new technology raises $t_{j}$ by one and the level of productivity $B_{j}^{t_{j}}$ by $\varepsilon>1$. Hence,

$$
B_{j}^{t_{j}}=B_{j}^{0} \varepsilon^{t_{j}}
$$

Noting (3)-(6) and denoting the expectations operation by $E$, we obtain the average growth rate of the average productivity $B$ in the stationary state as ${ }^{6}$

$$
g \doteq \sum_{j=1}^{J} E\left[\log B^{t_{j}+1,\left\{t_{k \neq j}\right\}}-\log B^{\left\{t_{k}\right\}}\right]
$$

\footnotetext{
${ }^{6}$ For this, see Aghion and Howitt (1998), p. 59.
} 


$$
\begin{aligned}
& \approx \sum_{j=1}^{J} E\left\{\left(\frac{B_{j}}{B} \frac{\partial \mathcal{B}}{\partial B_{j}}\right)^{\left\{t_{k}\right\}}\left[\log B_{j}^{t_{j}+1}-\log B_{j}^{t_{j}}\right]\right\} \\
& =(\log \varepsilon) \sum_{j=1}^{J} E\left[\left(\frac{B_{j}}{B} \frac{\partial \mathcal{B}}{\partial B_{j}}\right)^{\left\{t_{k}\right\}} \Lambda_{j}\right]=(\log \varepsilon) \frac{\lambda}{J} \sum_{j=1}^{J} l_{j} E\left[\left(\frac{B_{j}^{t_{j}}}{B^{\left\{t_{k}\right\}}}\right)^{1-\gamma}\right] .
\end{aligned}
$$

Because the dynamics of the model would be excessively complicated with asymmetry among the regions, we focus on a stationary state in which at some time $t=0$ the productivity is uniform in the whole economy, $B_{j}^{0}=B^{0}$ for all $j$. There is then perfect symmetry throughout all regions $j, n_{j}=n$ and $l_{j}=l$ for all $j$, so that the productivity parameters $B_{j}$, the average productivity in the economy, $B$, and aggregate consumption (4) grow on the average at the same rate (7). In Appendix A, we approximate

$$
E\left[\left(B_{j} / B\right)^{1-\gamma}\right]_{l_{k}=l, B_{k}^{0}=B^{0}} \approx 1 \text { for all } j,
$$

Noting this and (7), we obtain that on the average the growth rate of the economy is in fixed proportion $(\log \varepsilon) \lambda$ to the average level of $R \& D, l$ :

$$
\left.\left.g\right|_{l_{k}=l, B_{j}^{0}=B^{0}} \approx(\log \varepsilon) \frac{\lambda}{J} \sum_{j=1}^{J} l_{j}\right|_{l_{k}=l, B_{k}^{0}=B^{0}}=(\log \varepsilon) \lambda l .
$$

\section{Production}

We denote the price for the consumption good by $P$ and normalize aggregate consumption expenditure $P C$ at unity:

$$
P=1 / C
$$

Because in the households' preferences the decisions on the distribution of expenditures throughout all goods at each moment of time are separable from the decisions on the distribution of expenditures over time, in equilibrium the price $p_{j}$ must be equal to the marginal product $P\left(\partial C / \partial y_{j}\right)$ for each good $j$. Noting (1) and (10), this condition takes the form

$$
p_{j}=P \frac{\partial C}{\partial y_{j}}=P\left(\frac{C}{J y_{j}}\right)^{\gamma}=J^{-\gamma} C^{\gamma-1} y_{j}^{-\gamma} .
$$


There is one capitalist in each region $j$ (hereafter capitalist $j$ ) who owns producer $j$. Because his decisions on production are separable from his decisions on investment, which affect the productivity level $B_{j}$, we can assume that each producer $j$ maximizes its profit by employment $n_{j}$ for given $B_{j}$.

Each producer $j$ behaves in Cournot manner, taking the others' output levels $y_{k}(k \neq j)$ as given. ${ }^{7}$ It estimates the elasticity of the demand for its product in the vicinity of the equilibrium. With the assumption that at time $t=0$ the productivity is uniform in the economy, $B_{j}^{0}=B^{0}$, in equilibrium $n_{k}=n$ and $l_{k}=l$ holds for all $k$. Noting this, (1), (2), (4), (8) and (11), producer $j$ approximates the inverse of the anticipated price elasticity of demand for its output $j$ as follows:

$$
\begin{aligned}
\phi(J, \varphi) & \doteq-\left[\frac{y_{j}}{p_{j}} \frac{d p_{j}}{d y_{j}}\right]_{n_{k}=n, l_{k}=l, B_{k}^{0}=B^{0}}=-\left[\frac{y_{j}}{p_{j}}\left(\frac{\partial p_{j}}{\partial y_{j}}+\frac{\partial p_{j}}{\partial C} \frac{\partial C}{\partial y_{j}}\right)\right]_{n_{k}=n, l_{k}=l, B_{k}^{0}=B^{0}} \\
& =\gamma+(1-\gamma)\left[\frac{y_{j}}{C} \frac{\partial C}{\partial y_{j}}\right]_{n_{k}=n, l_{k}=l, B_{k}^{0}=B^{0}} \\
& =\gamma+\frac{1-\gamma}{J}\left[\left(\frac{B_{j}}{B}\right)^{1-\gamma}\right]_{l_{k}=l, B_{k}^{0}=B^{0}} \\
& \approx \gamma+(1-\gamma) / J \text { with } \partial \phi / \partial J<0 \text { and } \partial \phi / \partial \gamma>0
\end{aligned}
$$

In this model, the inverse of the elasticity of substitution between any pair of the goods, $\gamma$, characterizes the degree of competition in the goods market. The smaller $\gamma$, the higher the profits.

We denote the wage in region $j$ by $w_{j}$. Producer $j$ maximizes its profit $\pi_{j} \doteq p_{j} y_{j}-w_{j} n_{j}$ by its input $n_{j}$, given the production function (2) and the anticipated elasticity (12). This and (11) yield the equilibrium conditions

$$
\begin{aligned}
& w_{j}=\left[p_{j}+y_{j}\left(d p_{j} / d y_{j}\right)\right] B_{j}=(1-\phi) p_{j} B_{j}=(1-\phi) J^{-\gamma} C^{\gamma-1} n_{j}^{-\gamma} B_{j}^{1-\gamma}, \\
& \pi_{j}=p_{j} y_{j}-(1-\phi) w_{j} B_{j} n_{j}=\phi p_{j} y_{j}, \quad w_{j} n_{j} / \pi_{j}=1 / \phi-1 .
\end{aligned}
$$

${ }^{7}$ This is the simplest form of strategic interdependence between the producers. With slight complication, the same results could be extended for the more general case in which each producer $j$ anticipates the reaction of the others $k \neq j$ by $d y_{k} / d y_{j}=\varphi y_{k} / y_{j}$ for $k \neq j$, where $\varphi<1$ is a constant. 
Because here a producer receives the constant share $\phi$ of value added, we use $\phi$ as a measure of producer market power. From (12) it follows that increased competition (i.e. a smaller $\gamma$ ) or a bigger size $J$ of the economy decrease producer market power $\phi$.

\section{Capitalists}

Capitalist $j$ earns the profit $\pi_{j}$. His budget constraint is given by

$$
\pi_{j}=P C_{j}+w_{j} l_{j}
$$

where $C_{j}$ is consumption, $P$ the consumption price and $w_{j} l_{j}$ investment expenditure (= saving). During a short time interval $d \theta$, there is a change in technology with probability $\Lambda_{j} d \theta$, and no change with probability $1-\Lambda_{j} d \theta$, where $\Lambda_{j}$ is given by (5). Capitalist $j$ maximizes the present value of his consumption flow subject to the budget constraint (14) and technological change by his investment in $\mathrm{R} \& \mathrm{D}, l_{j}$, given the wage $w_{j}$ and the consumption price $P$ (or aggregate consumption $C=1 / P$ ). With Ramsey preferences, the value of capitalist $j$ 's optimal program at time $T$ is given by

$$
\begin{aligned}
& \Omega\left(t_{j}, w_{j}, \pi_{j}, P\right)=\max _{l_{j}} E \int_{T}^{\infty} C_{j}^{\sigma} e^{-\rho(\theta-T)} d \theta \\
& \text { with } 0<\sigma<1, \rho>0 \text { and } C_{j}=\left(\pi_{j}-w_{j} l_{j}\right) / P,
\end{aligned}
$$

where $\theta$ is time, $E$ the expectation operator, $\rho$ the rate of time preference and $1 /(1-\sigma)$ is the constant rate of relative risk aversion.

In Appendix B, we show that capitalist $j$ 's optimization leads to the following two results. First, capitalist $j$ 's propensity to consume, $c_{j}$, is negatively associated with his investment in $\mathrm{R} \& \mathrm{D}, l_{j}$ :

$$
\frac{P C_{j}}{\pi_{j}}=c_{j}=c\left(l_{j}\right), \quad c^{\prime}=\frac{\left(c_{j}-1\right) c_{j} \rho / l_{j}}{\rho+\left[1-\varepsilon^{(1-\gamma) \sigma}\right] \lambda l_{j}}<0, \quad \rho+\left[1-\varepsilon^{(1-\gamma) \sigma}\right] \lambda l_{j}>0 .
$$


When the capitalist consumes more, he saves less and invests less in R\&D. Second, employment in production is determined by

$$
n_{j}=\frac{(1 / \phi-1) l_{j}}{1-c_{j}}=\frac{(1 / \phi-1) l_{j}}{1-c\left(l_{j}\right)} \doteq n\left(l_{j}, \phi\right), \quad \frac{\partial n}{\partial \phi}<0 .
$$

Hence, for given $\mathrm{R} \& \mathrm{D} l_{j}$, higher producer market power $\phi$ increases the output price $p_{j}$ and decreases employment in production, $n_{j}$. If the propensity to consume, $c_{j}$, is kept constant, then income, saving and real investment in $\mathrm{R} \& \mathrm{D}, l_{j}$, are in fixed proportion to the scale $n_{j}$ of production. On the other hand, employment in $\mathrm{R} \& \mathrm{D}, l_{j}$, crowds out employment in production, $n_{j}$, through higher wages. Because of these two opposing effects, the employment in production $n_{j}$ and in $\mathrm{R} \& \mathrm{D} l_{j}$ are ambiguously associated.

\section{$5 \quad$ Wage bargaining}

All workers in region $j$ belong to the same union labelled $j$. In order to derive the union's preferences from workers' preferences, we make the following two assumptions, for tractability:

(a) Although the workers do not save, they (or the union leaders) have the same rate of time preference $\rho>0$ and the same rate of risk aversion $1 /(1-\sigma)$ as the capitalists.

(b) The workers have access to perfect unemployment insurance.

Assumption (a) ensures that workers' and capitalists' utilities grow at the same rate. Otherwise, there would be no stationary state in the model. Given assumption $(b)$, all workers in the same region behave as if there were only one worker. The violation of $(b)$ through taxation or a more complex system of unemployment insurance would affect income distribution among the working class. In the present study, such distributional aspects would excessively complicate the analysis and therefore they are left for future investigation. 
Union $j$ and capitalist $j$ bargain over the wage $w_{j}$. From assumptions $(a)$ and $(b)$ above it follows that the union's expected utility at time $T$ can be expressed by the representative workers' utility

$$
\mathcal{U}_{j}\left(l_{j}, \phi\right) \doteq \int_{T}^{\infty}\left[\left(n_{j}+l_{j}\right) w_{j} / P\right]^{\sigma} e^{-\rho(\theta-T)} d \theta
$$

where $\left(n_{j}+l_{j}\right) w_{J} / P$ is the representative worker's consumption (= real income). The capitalist's expected utility at time $T$ is given by

$$
\mathcal{F}_{j}\left(l_{j}, \phi\right) \doteq \int_{T}^{\infty} C_{j}^{\sigma} e^{-\rho(\theta-T)} d \theta
$$

The union and the capitalist, which operate within a single region, take the consumption price $P$ and aggregate consumption $C=1 / P$ as given. Noting (13), we obtain that both $\left(n_{j}+l_{j}\right) w_{j} / P$ and $C_{j}=c_{j} \pi_{j} / P$ grow at the same rate as $B_{j}^{1-\gamma}$. The parties' targets (18) and (19) can then be transformed into the following form: ${ }^{8}$

$$
\begin{aligned}
& \mathcal{U}_{j}\left(l_{j}, \phi\right)=\frac{B_{j}(T)^{(1-\gamma) \sigma}\left(n_{j}+l_{j}\right)^{\sigma} w_{j}^{\sigma}}{P^{\sigma} B_{j}^{(1-\gamma) \sigma}\left[\rho+\left(1-\varepsilon^{(1-\gamma) \sigma}\right) \lambda l_{j}\right]}, \\
& \mathcal{F}_{j}\left(l_{j}, \phi\right)=\frac{B_{j}(T)^{(1-\gamma) \sigma} c_{j}^{\sigma} \pi_{j}^{\sigma}}{P^{\sigma} B_{j}^{(1-\gamma) \sigma}\left[\rho+\left(1-\varepsilon^{(1-\gamma) \sigma}\right) \lambda l_{j}\right]} .
\end{aligned}
$$

Union $j$ (capitalist $j$ ) maximizes its expected utility $\mathcal{U}_{j}\left(\mathcal{F}_{j}\right)$ for given $P$ and $C$. Because there is one-to-one correspondence from $w_{j}$ to $l_{j}$ through (13) and (17), in this maximization $w_{j}$ can be replaced by $l_{j}$ as the control variable. The outcome of bargaining is then obtained through maximizing by $l_{j}$ the Generalized Nash Product $\mathcal{U}_{j}^{\alpha} \mathcal{F}_{j}^{1-\alpha}$, where the constant $\alpha \in(0,1)$ is relative union bargaining power. Through this maximization, we obtain in Appendix $\mathrm{C}$ that both relative union bargaining power $\alpha$ and producer market power $\phi$ promote R\&D:

$$
l_{j}=l(\alpha, \phi), \quad \partial l / \partial \alpha>0, \quad \partial l / \partial \phi>0 .
$$

\footnotetext{
${ }^{8}$ For this, see e.g. Aghion and Howitt (1998), p. 61.
} 
With higher union power $\alpha$, wages increase. With higher producer market power $\phi$, capitalists can escape from a greater proportion of wage increases through price increases. It is then easier for them to accept unions' wage claims and wages increase even further. With higher wages, capitalists have every incentive to increase the productivity of labour through R\&D. With more $R \& D$, there will be more innovations and a higher growth rate.

\section{A common market}

Let the economy under consideration be a common market which can expand smoothly by accepting new regions as its members. We assume that the new members have access to the same technology and must adopt the same institutions as the old members, so that economic integration can be characterized by the increase in the size $J$ of the common market. In such a case, the government of the common market can use $J$ as a policy instrument.

We define the government's target as the weighed average of the workers' average utility $(1 / J) \sum_{j} \mathcal{U}_{j}$ and capitalists' average utility $(1 / J) \sum_{j} \mathcal{F}_{j}$ :

$$
\mathcal{W}=\frac{1}{J} \sum_{j} \mathcal{U}_{j}+\frac{\xi}{J} \sum_{j} \mathcal{F}_{j},
$$

where the constant $\xi \in[0, \infty)$ characterizes the capitalists' political influence. There is a pure 'labour' government in the common market for $\xi=0$ and a pure 'capitalist' government for $\xi \rightarrow \infty$.

On the assumption that the number of regions, $J$, is large, we transform in Appendix D the government's welfare function (22) into the following form:

$$
\mathcal{W}=\int_{T}^{\infty} \varepsilon^{\sigma t} \chi(l, \phi, \xi) e^{-\rho(\theta-T)} d \theta, \quad \frac{\partial \chi}{\partial \phi}<0, \quad \frac{\partial^{2} \chi}{\partial \phi \partial \xi}<0, \quad \frac{\partial^{2} \chi}{\partial l \partial \xi}<0 .
$$

In order to examine optimal integration, we assume that the government maximizes its target (23) by the size $J$ of the common market. Given (12), the government can equivalently maximize (23) by $\phi$. In order to examine the effect of relative union bargaining power, we assume that also $\alpha$ is the 
government's policy instrument. Denoting the value of the state of technology $t$ for the government by $\Upsilon(t, \alpha, \phi)$, noting (5), (21) and (23), we obtain the Bellman equation for the government's maximization as follows:

$$
\begin{aligned}
& \rho \Upsilon(t)=\max _{\phi, \alpha} Q(t, \phi, \alpha), \text { where } \\
& Q(t, \phi, \alpha, \xi) \doteq R(t, l(\alpha, \phi), \phi, \xi) \doteq \varepsilon^{\sigma t} \chi(l, \phi, \xi)+\lambda l[\Upsilon(t+1)-\Upsilon(t)] .
\end{aligned}
$$

Assume that, for some unspecified reason, the government can optimally determine relative union bargaining power $\alpha$. Noting (21), the government can then fully control the level of $\mathrm{R} \& \mathrm{D}, l$, by $\alpha$. The first-order condition for $\alpha$ corresponding to the Bellman equation (24) takes then the form $\partial R / \partial l=$ $\varepsilon^{\sigma t} \partial \chi / \partial l+\lambda[\Upsilon(t+1)-\Upsilon(t)]=0$. This equation defines $l$ as a function of $\phi$ and $\xi$. Differentiating it totally, and noting (23) and the second-order condition $\partial^{2} R / \partial l^{2}=\varepsilon^{\sigma t} \partial^{2} \chi / \partial l^{2}<0$, we obtain $\partial^{2} \chi / \partial l^{2}<0$ and

$$
\frac{\partial l}{\partial \xi}=-\frac{\partial^{2} \chi}{\partial l \partial \xi} / \frac{\partial^{2} \chi}{\partial l^{2}}<0
$$

Because $\partial l / \partial \alpha>0$ by (21), R\&D can be promoted (hampered) by increasing (decreasing) $\alpha$. This and (25) yield the following sub-result:

Proposition 1 If the government can optimally set relative union bargaining power $\alpha$, then the optimal level of $R \& D$ for the government of the common market is given by $l^{*}(\phi, \xi) \doteq \arg \max _{l} R(t, l, \phi, \xi)$. The government should discourage (encourage) $R \& D l$ through decreasing (increasing) relative union bargaining power $\alpha$ for $l>l^{*}\left(l<l^{*}\right)$. The stronger the capitalists' political influence (i.e. the higher $\xi$ ), the lower the optimal level of $R \& D, \partial l^{*} / \partial \xi<0$.

Technological change due to $R \& D$ increases wages and profits in the same proportion. Because R\&D means wages for workers but costs for capitalists, the capitalists prefer a lower level of R\&D than the workers, $\partial l^{*} / \partial \xi<0$. 


\section{Economic integration}

In this section, we assume that relative union bargaining power $\alpha$ is exogenously given. Consider first the case where union power is optimal $\partial Q / \partial \alpha=$ $(\partial R / \partial l) \partial l / \partial \alpha=0$ or excessive $\partial Q / \partial \alpha=(\partial R / \partial l) \partial l / \partial \alpha<0$ for the government's viewpoint. Noting this, (23), (24) and proposition (1), we obtain $\partial l / \partial \alpha>0, \partial l / \partial \phi>0, \partial R / \partial l \leq 0, l \geq l^{*} \doteq \arg \max _{l} R(t, l, \phi, \xi)$ and

$$
\frac{\partial Q}{\partial \phi}=\frac{\partial R}{\partial \phi}+\frac{\partial R}{\partial l} \frac{\partial l}{\partial \phi} \leq \frac{\partial R}{\partial \phi}=\varepsilon^{\sigma t} \frac{\partial \chi}{\partial \phi}<0 .
$$

This means that the government of the common market increases the size $J$ of the common market to decrease $\phi[$ Cf. (12)]. We conclude:

Proposition 2 If relative union bargaining power $\alpha$ is high enough for $l \geq l^{*}(\phi, \xi)$ to hold, then the common market accepts new members.

When high union power generates excessive economic growth $l \geq l^{*}$, all growth-hampering measures are welfare enhancing. By taking in new members, the government increases the number of producers, decreases producer market power $\phi$ and thereby slows down economic growth.

Next, consider the remaining case where union power is sub-optimal, $\partial Q / \partial \alpha=(\partial R / \partial l) \partial l / \partial \alpha>0$ and $\partial R / \partial l>0$. The first-order and secondorder conditions for $\phi$ corresponding to (24) is then given by

$$
\frac{\partial Q}{\partial \phi}=\frac{\partial R}{\partial \phi}+\frac{\partial R}{\partial l} \frac{\partial l}{\partial \phi}=\varepsilon^{\sigma t} \frac{\partial \chi}{\partial \phi}+\frac{\partial R}{\partial l} \frac{\partial l}{\partial \phi}=0, \quad \frac{\partial^{2} Q}{\partial \phi^{2}}<0 .
$$

From this equation we can solve for the optimal level $\phi^{*}$ of producer market power $\phi$ and consequently, for the optimal size $J^{*}$ of the common market. Noting (12), (23), (24) and (26), we obtain the following functions:

$$
\begin{aligned}
& \frac{\partial^{2} Q}{\partial \phi \partial \xi}=\varepsilon^{\sigma t} \frac{\partial^{2} \chi}{\partial \phi \partial \xi}+\frac{\partial^{2} \chi}{\partial l \partial \xi} \frac{\partial l}{\partial \phi}<0, \quad \phi^{*}(\alpha, \xi), \quad \frac{\partial \phi^{*}}{\partial \xi}=-\frac{\partial^{2} Q}{\partial \phi \partial \xi} / \frac{\partial^{2} Q}{\partial \phi^{2}}<0, \\
& J^{*}(\alpha, \xi, \gamma), \frac{\partial J^{*}}{\partial \xi}=\frac{\partial \phi^{*}}{\partial \xi} / \frac{\partial \phi}{\partial J}>0, \quad \frac{\partial J^{*}}{\partial \gamma}=-\frac{\partial \phi}{\partial \xi} / \frac{\partial \phi}{\partial J}>0 .
\end{aligned}
$$

The last function can be rephrased as follows: 
Proposition 3 If relative union bargaining power $\alpha$ is low enough for $l<l^{*}(\phi, \xi)$ to hold, then there is an upper limit $J^{*}(\alpha, \gamma, \xi)$ to the size $J$ of the common market. The limit $J^{*}$ is the higher, the less there is competition in the goods market (i.e. the bigger $\gamma$ ), $\partial J^{*} / \partial \gamma>0$, or the stronger the capitalists' political influence (i.e. the bigger $\xi$ ), $\partial J^{*} / \partial \xi>0$.

Proposition 3 can be explained as follows. Assume that union power is not so high that it would generate excessive growth, $l \leq l^{*}$. Economic integration increases the number of producers and decreases producer market power $\phi$. This lowers prices and raises employment, current real income and welfare. On the other hand, with weaker market power capitalists cannot as easily escape from wage increases through price increases and union-capitalist bargaining results in lower wages. With lower wages, the capitalists have less incentives to invest in $R \& D$, the growth rate falls and welfare decreases. The common market integrates new regions as long as the current-income effect outweighs the growth effect. Because competition in the goods market and economic integration are strategic substitutes, the decrease in the former should increase the latter at the optimum. Technological change due to R\&D increases wages and profits in the same proportion, but R\&D incudes income for workers but costs for capitalists. Hence, which stronger political influence by capitalists, the government cares less about the welfare-diminishing growth effect of integration and accepts more regions in the common market.

From propositions 2 and 3 it follows that workers (or the 'labour' government with $\xi=0)$ are willing to extend the common market only if $l<l^{*}(\phi, 0)$ and $J<J^{*}(\alpha, \gamma, 0)$ hold, but the government is willing to do so only if $l<l^{*}(\phi, \xi)$ and $J<J^{*}(\alpha, \gamma, \xi)$ hold. This yields the following corollary:

Proposition 4 Economic integration benefits capitalists more than workers. If the common market grows at a "medium" rate, $l \in\left[l^{*}(\phi, \xi), l^{*}(\phi, 0)\right)$, and if it is of "medium" size $J \in\left(J^{*}(\alpha, \gamma, 0), J^{*}(\alpha, \gamma, \xi)\right]$, then it takes new members, although this harms workers. 
At low growth rates, nobody is willing to slow down growth even further though integration. At high growth rates, even workers are willing to substitute current income for growth though integration. Hence, at the 'medium' rates of growth, the capitalists are but the workers aren't willing to integrate.

\section{Conclusions}

This paper examines a common market with a large number of regions, each producing a different good. The market expands by integrating new regions. Capitalists can improve their productivity through investment in R\&D. Profits are the higher, the smaller is the elasticity of substitution between any pair of the goods. Production and R\&D employ workers who are unionized. Both workers and capitalists can influence the government which decides on new members for the common market. The main findings are the following.

Both relative union bargaining power and producer market power promote $R \& D$ and economic growth. With stronger unions, wages increase. With higher producer market power, capitalists can escape from a greater proportion of wage increases through price increases. Hence, it is easier for them to accept unions' wage claims and wages increase even further. With higher wages, capitalists have every incentive to improve the productivity of labour through $R \& D$. Increased $R \& D$ promotes economic growth.

When high union power generates excessive economic growth, all growthhampering measures are welfare enhancing. By taking in new members, the government increases the number of producers, decreases producer market power which slows down economic growth. Otherwise, there is an upper limit to the size of the common market. This limit is the higher, the less there is competition in the goods market or the stronger the capitalists' influence on the government. This can be explained as follows.

Because economic integration increases the number of producers, it weakens a single producer's market power. This decreases prices and increases 
employment, current real income and welfare. On the other hand, with lower producer market power capitalists cannot as easily escape from wage increases through price increases and union-capitalist bargaining results in lower wages. With lower wages, the capitalists have less incentives to invest in $\mathrm{R} \& \mathrm{D}$, the growth rate falls and welfare decreases. The common market accepts new members as long as the welfare-enhancing current-income effect dominates over the welfare-diminishing growth effect. Because product market competition and economic integration both diminish producer market power, the decrease in the former increases the latter at the optimum.

Economic integration benefits capitalists more than workers. Because technological change due to $\mathrm{R} \& \mathrm{D}$ increases wages and profits in the same proportion, but R\&D as such means wages for workers but costs for capitalists, the growth-diminishing effect of integration harms capitalists less than workers. Hence, when the capitalists have stronger political influence, the government cares less about the growth-diminishing effect of integration and accepts more regions in the common market.

If a common market of "medium" size grows at a "medium" rate, then it takes new members, although this harms workers. At low growth rates, nobody is willing to slow down growth even further though integration. At high growth rates, even workers are willing to substitute current income for growth though integration. Hence, at the 'medium' rates of growth, the capitalists are willing but the workers are unwilling to integrate.

\section{Appendix}

\section{A. The equation (8)}

Noting (3) and (5)-(7), we obtain the average growth rate of the term 
$\left(B_{j} / B\right)^{1-\gamma}$ in the stationary state with $l_{j}=l$ for all $j$ as follows: ${ }^{9}$

$$
\begin{aligned}
\sum_{k=1}^{J} E & \left\{\log \left[\left(\frac{B_{j}}{B}\right)^{1-\gamma}\right]^{t_{k}+1,\left\{t_{\ell \neq k}\right\}}-\log \left[\left(\frac{B_{j}}{B}\right)^{1-\gamma}\right]^{\left\{t_{\ell}\right\}}\right\} \\
& =(1-\gamma) \sum_{k=1}^{J} E\left\{\log \left[\left(\frac{B_{j}}{B}\right)\right]^{t_{k}+1,\left\{t_{\ell \neq k}\right\}}-\log \left[\left(\frac{B_{j}}{B}\right)\right]^{\left\{t_{\ell}\right\}}\right\} \\
& =(1-\gamma)\left\{E\left[\log B_{j}^{t_{j}+1}-\log B_{j}^{t_{j}}\right]-\sum_{k=1}^{J} E\left[\log B^{t_{k}+1,\left\{t_{\ell \neq k}\right\}}-\log B^{\left\{t_{\ell}\right\}}\right]\right\} \\
& =(1-\gamma) \lambda(\log \varepsilon)\left\{l_{j}-\frac{1}{J} \sum_{k=1}^{J} l_{k} E\left[\left(\frac{B_{k}^{t_{k}}}{B^{\left\{t_{\ell}\right\}}}\right)^{1-\gamma}\right]\right\} \\
& =(1-\gamma) \lambda(\log \varepsilon) l\left\{1-\frac{1}{J} \sum_{k=1}^{J} E\left[\left(\frac{B_{k}^{t_{k}}}{B^{\left\{t_{\ell}\right\}}}\right)^{1-\gamma}\right]\right\} \equiv 0,
\end{aligned}
$$

where $E$ is the expectation operator. This shows that the term $\left(B_{j} / B\right)^{1-\gamma}$ has no trend. Noting this and $B_{j}^{0}=B^{0}$, we obtain (8).

B. The functions (16) and (17)

From (6) and (13) it follows that

$$
\pi_{j}^{t_{j}+1} / \pi_{j}^{t_{j}}=\left(B_{j}^{t_{j}+1} / B_{j}^{t_{j}}\right)^{1-\gamma}=\varepsilon^{1-\gamma} .
$$

The Bellman equation corresponding to (15) is given by ${ }^{10}$

$$
\rho \Omega\left(t_{j}, w_{j}\right)=\max _{l_{j}}\left\{C_{j}^{\sigma}+\Lambda_{j}\left[\Omega\left(t_{j}+1, w_{j}, \pi_{j}, P\right)-\Omega\left(t_{j}, w_{j}, \pi_{j}, P\right)\right]\right\},
$$

where $\Lambda_{j}=\lambda l_{j}$ and $C_{j}=\left(\pi_{j}-w_{j} l_{j}\right) / P$. The first order condition for investment $l_{j}$ is given by

$$
\lambda\left[\Omega\left(t_{j}+1, w_{j}, \pi_{j}, P\right)-\Omega\left(t_{j}, w_{j}, \pi_{j}, P\right)\right]=\frac{\sigma w_{j}}{P} C_{j}^{\sigma-1}
$$

We try the solution $\Omega=C_{j}^{\sigma} / r_{j}=\left(c_{j} \pi_{j}\right)^{\sigma} / r_{j}$, in which capitalist $j$ 's propensity to consume, $c_{j} \doteq P C_{j} / \pi_{j} \in[0,1]$, and subjective discount factor $r_{j}>0$

\footnotetext{
${ }^{9}$ For this, see Aghion and Howitt (1998), p. 59

${ }^{10}$ Cf. Dixit and Pindyck (1994), Wälde (1999).
} 
are independent of income $\pi_{j}$. Given (27), we obtain

$$
\begin{aligned}
\widetilde{\Omega} & \doteq \Omega\left(t_{j}+1, w_{j}, \pi_{j}, P\right)=\left(c_{j} \pi_{j}^{t_{j}+1}\right)^{\sigma} / r_{j}=\varepsilon^{(1-\gamma) \sigma}\left(c_{j} \pi_{j}^{t_{j}}\right)^{\sigma} / r_{j} \\
& =\varepsilon^{(1-\gamma) \sigma} \Omega\left(t_{j}, w_{j}, \pi_{j}, P\right) .
\end{aligned}
$$

Inserting this and $\Omega=C_{j}^{\sigma} / r_{j}$ into (28) yield

$$
\begin{aligned}
& \rho=C_{j}^{\sigma} / \Omega+\lambda l_{j}[\widetilde{\Omega} / \Omega-1]=r_{j}+\left[\varepsilon^{(1-\gamma) \sigma}-1\right] \lambda l_{j}, \\
& r_{j}=\rho+\left[1-\varepsilon^{(1-\gamma) \sigma}\right] \lambda l_{j}>0 .
\end{aligned}
$$

From $P C_{j}=c_{j} \pi_{j}$ and (14) it follows that

$$
w_{j} l_{j}=\left(1-c_{j}\right) \pi_{j}=\left(1 / c_{j}-1\right) P C_{j} .
$$

Inserting $\widetilde{\Omega}=\varepsilon^{(1-\gamma) \sigma} \Omega, \Omega=C_{j}^{\sigma} / r_{j}$, (30) and (31) into (29), we obtain

$$
\begin{aligned}
{\left[\varepsilon^{(1-\gamma) \sigma}-1\right] \lambda } & =\lambda\left(\frac{\widetilde{\Omega}}{\Omega}-1\right)=\frac{\sigma w_{j}}{P \Omega} C_{j}^{\sigma-1}=\frac{\sigma w_{j} r_{j}}{P C_{j}}=\sigma \frac{r_{j}}{l_{j}}\left(\frac{1}{c_{j}}-1\right) \\
& =\sigma\left\{\frac{\rho}{l_{j}}+\left[1-\varepsilon^{(1-\gamma) \sigma}\right] \lambda\right\}\left(\frac{1}{c_{j}}-1\right) .
\end{aligned}
$$

This equation defines the function

$$
\frac{P C_{j}}{\pi_{j}}=c_{j}=c\left(l_{j}\right), \quad c^{\prime}=-\frac{\left(1-c_{j}\right) c_{j} \rho / l_{j}}{\rho+\left[1-\varepsilon^{(1-\gamma) \sigma}\right] \lambda l_{j}}<0 .
$$

Noting (13), (31) and (32), we obtain

$$
n_{j}=\left(\frac{1}{\phi}-1\right) \frac{\pi_{j}}{w_{j}}=\frac{(1 / \phi-1) l_{j}}{1-c_{j}}=\frac{(1 / \phi-1) l_{j}}{1-c\left(l_{j}\right)} \doteq n\left(l_{j}, \phi\right), \quad \frac{\partial n}{\partial \phi}<0 .
$$

C. The function (21)

Given (13), (16), (17) and (20), the outcome of bargaining is obtained through maximizing by $l_{j}$ the following increasing transformation of the Generalized Nash product $\mathcal{U}_{j}^{\alpha} \mathcal{F}_{j}^{1-\alpha}$ :

$\Gamma_{j}\left(l_{j}, C, \alpha\right) \doteq(1 / \sigma) \log \left[\mathcal{U}_{j}^{\alpha} \mathcal{F}_{j}^{1-\alpha}\right]=(1 / \sigma)\left[\alpha \log \mathcal{U}_{j}+(1-\alpha) \log \mathcal{F}_{j}\right]$ 


$$
\begin{aligned}
=\alpha \log \left[\left(n_{j}+l_{j}\right) w_{j} B_{j}^{\gamma-1}\right]+(1-\alpha) \log \left[c_{j} \pi_{j} B_{j}^{\gamma-1}\right] & \\
& -(1 / \sigma) \log \left\{\rho+\left[1-\varepsilon^{(1-\gamma) \sigma}\right] \lambda l_{j}\right\}+\Delta \\
=\alpha \log \left(1+l_{j} / n_{j}\right)+\alpha \log \left[w_{j} n_{j} B_{j}^{\gamma-1}\right]+(1-\alpha) \log \left[c_{j} w_{j} n_{j} B_{j}^{\gamma-1}\right] & \\
& -(1 / \sigma) \log \left\{\rho+\left[1-\varepsilon^{(1-\gamma) \sigma}\right] \lambda l_{j}\right\}+\Delta \\
=\alpha \log \left(1+l_{j} / n_{j}\right)+(1-\alpha) \log c_{j}+\log \left[w_{j} n_{j} B_{j}^{\gamma-1}\right] & \\
& -(1 / \sigma) \log \left\{\rho+\left[1-\varepsilon^{(1-\gamma) \sigma}\right] \lambda l_{j}\right\}+\Delta \\
=\alpha \log \left(1+l_{j} / n_{j}\right)+(1-\alpha) \log c_{j}+(1-\gamma) \log n_{j} & -(1 / \sigma) \log \left\{\rho+\left[1-\varepsilon^{(1-\gamma) \sigma}\right] \lambda l_{j}\right\}+\Delta \\
=\alpha \log & {\left[1+\frac{1-c\left(l_{j}\right)}{1 / \phi-1}\right]+(1-\alpha) \log c\left(l_{j}\right)+(1-\gamma)\left\{\log l_{j}-\log \left[1-c\left(l_{j}\right)\right]\right\} } \\
& -(1 / \sigma) \log \left\{\rho+\left[1-\varepsilon^{(1-\gamma) \sigma}\right] \lambda l_{j}\right\}+\Delta
\end{aligned}
$$

with $\rho+\left[1-\varepsilon^{(1-\gamma) \sigma}\right] \lambda l_{j}>0$,

where $\Delta$ denotes terms that are independent of $l_{j}$. Noting (33), we obtain the first-order condition

$$
\begin{aligned}
\frac{\partial \Gamma_{j}}{\partial l_{j}}=(1-\alpha) \frac{c^{\prime}\left(l_{j}\right)}{c\left(l_{j}\right)}-\frac{\alpha c^{\prime}\left(l_{j}\right)}{1 / \phi-c\left(l_{j}\right)}+(1-\gamma)\left[\frac{1}{l_{j}}+\frac{c^{\prime}\left(l_{j}\right)}{1-c\left(l_{j}\right)}\right] \\
+\frac{\left[\varepsilon^{(1-\gamma) \sigma}-1\right] \lambda / \sigma}{\rho+\left[1-\varepsilon^{(1-\gamma) \sigma}\right] \lambda l_{j}}=0,
\end{aligned}
$$

which defines the function $l_{j}=l(\alpha, \phi, b)$. Noting

$$
\frac{\partial^{2} \Gamma_{j}}{\partial l_{j} \partial \alpha}=-\frac{c^{\prime}}{c}-\frac{c^{\prime}}{1 / \phi-c}>0, \quad \frac{\partial^{2} \Gamma_{j}}{\partial l_{j} \partial \phi}=-\frac{\alpha c^{\prime}}{(1-\phi c)^{2}}>0,
$$

and the second-order condition $\partial^{2} \Gamma_{j} / \partial l_{j}^{2}<0$, we obtain

$$
\frac{\partial l}{\partial \alpha}=-\frac{\partial^{2} \Gamma_{j}}{\partial l_{j} \partial \alpha} / \frac{\partial^{2} \Gamma_{j}}{\partial l_{j}^{2}}>0, \quad \frac{\partial l}{\partial \phi}=-\frac{\partial^{2} \Gamma_{j}}{\partial l_{j} \partial \phi} / \frac{\partial^{2} \Gamma_{j}}{\partial l_{j}^{2}}>0 .
$$


D. The results (23)

Noting $n_{k}=n,(1),(2),(3),(8),(10)$ and (13), we obtain

$$
\begin{aligned}
& C=J\left(\frac{1}{J} \sum_{k=1}^{J} y_{k}^{1-\gamma}\right)^{1 /(1-\gamma)}={ }^{(2)} J\left(\frac{1}{J} \sum_{k=1}^{J} n_{k}^{1-\gamma} B_{k}^{1-\gamma}\right)^{1 /(1-\gamma)} \\
&=n J\left(\frac{1}{J} \sum_{k=1}^{J} B_{k}^{1-\gamma}\right)^{1 /(1-\gamma)}=n J B \\
& \frac{1}{J} \sum_{j=1}^{J}\left(\frac{w_{j} n_{j}}{P B}\right)^{\sigma}={ }^{(10)} \frac{1}{J} \sum_{j=1}^{J}\left(w_{j} n_{j} \frac{C}{B}\right)^{\sigma}=\frac{n^{\sigma}}{J} \sum_{j=1}^{J}\left(J w_{j} n_{j}\right)^{\sigma} \\
&=(1-\phi)^{\sigma} \frac{n^{\sigma}}{J} \sum_{j=1}^{J}\left(\frac{J n_{j} B_{j}}{C}\right)^{\sigma}={ }^{n_{j}=n}(1-\phi)^{\sigma} \frac{n^{\sigma}}{J} \sum_{j=1}^{J}\left(\frac{J n B_{j}}{C}\right)^{\sigma} \\
& \quad=(1-\phi)^{\sigma} \frac{n^{\sigma}}{J} \sum_{j=1}^{J}\left(\frac{B_{j}}{B}\right)^{(1-\gamma) \sigma} \approx(1-\phi)^{\sigma} n^{\sigma} \text { if } J \text { is large, } \\
& \frac{1}{J} \sum_{j=1}^{J}\left(\frac{\pi_{j}}{P B}\right)^{\sigma}=\left(\frac{\phi}{1-\phi}\right)^{\sigma} \frac{1}{J} \sum_{j=1}^{J}\left(\frac{w_{j} n_{j}}{P B}\right)^{\sigma} n^{\sigma} \approx \phi^{\sigma} n^{\sigma} \text { if } J \text { is large. }
\end{aligned}
$$

By choosing $B(0)=1$, we obtain $B=\varepsilon^{t}$ by (6). From $B=\varepsilon^{t}$, (10), (16), (17), (18), (19), (22), (34) and (35) it follows that

$$
\begin{aligned}
\mathcal{W}=\sum_{j} \frac{1}{J} \mathcal{U}_{j}+\frac{\xi}{J} \sum_{j} \mathcal{F}_{j}=\int_{T}^{\infty} \varepsilon^{\sigma t} \chi(l, \phi, \xi) e^{-\rho(\theta-T)} d \theta \text { with } \\
\chi(l, \phi, \xi)=\frac{1}{B^{\sigma}}\left\{\frac{1}{J} \sum_{j=1}^{J}\left[\frac{w_{j}}{P}\left(n_{j}+l_{j}\right)\right]^{\sigma}+\frac{\xi}{J} \sum_{j=1}^{J} C_{j}^{\sigma}\right\} \\
=\frac{1}{B^{\sigma}}\left[\frac{1}{J} \sum_{j=1}^{J}\left(1+\frac{l_{j}}{n_{j}}\right)^{\sigma}\left(\frac{w_{j} n_{j}}{P}\right)^{\sigma}+c^{\sigma} \frac{\xi}{J} \sum_{j=1}^{J}\left(\frac{\pi_{j}}{P}\right)^{\sigma}\right] \\
=\frac{1}{J} \sum_{j=1}^{J}\left(1+\frac{l_{j}}{n_{j}}\right)^{\sigma}\left(\frac{w_{j} n_{j}}{P B}\right)^{\sigma}+c^{\sigma} \frac{\xi}{J} \sum_{j=1}^{J}\left(\frac{\pi_{j}}{P B}\right)^{\sigma} \\
=\left(1+\frac{1-c}{1 / \phi-1}\right)^{\sigma} \frac{1}{J} \sum_{j=1}^{J}\left(\frac{w_{j} n_{j}}{P B}\right)^{\sigma}+c^{\sigma} \frac{\xi}{J} \sum_{j=1}^{J}\left(\frac{\pi_{j}}{P B}\right)^{\sigma} \\
\approx n^{\sigma}\left[\left(1+\frac{1-c}{1 / \phi-1}\right)^{\sigma}(1-\phi)^{\sigma}+c^{\sigma} \xi \phi^{\sigma}\right]
\end{aligned}
$$




$$
\begin{aligned}
& =n^{\sigma}\left[\left(\frac{1 / \phi-c}{1 / \phi-1}\right)^{\sigma}(1-\phi)^{\sigma}+\xi c^{\sigma} \phi^{\sigma}\right]=n^{\sigma}\left[(1-\phi c)^{\sigma}+\xi c(l)^{\sigma} \phi^{\sigma}\right] \\
& =\phi^{\sigma} n(l, \phi)^{\sigma}\left\{\left[\frac{1}{\phi}-c(l)\right]^{\sigma}+\xi c(l)^{\sigma}\right\} \\
& =\phi^{\sigma} n(l, \phi)^{\sigma} c(l)^{\sigma}\left\{\left[\frac{1}{\phi c(l)}-1\right]^{\sigma}+\xi\right\} \\
& =\left[\frac{(1-\phi) l}{1 / c(l)-1}\right]^{\sigma}\left\{\left[\frac{1}{\phi c(l)}-1\right]^{\sigma}+\xi\right\} .
\end{aligned}
$$

Given (16) and (36), we obtain

$$
\begin{aligned}
& \frac{\partial \chi}{\partial \phi}<0, \quad \frac{\partial \chi}{\partial \xi}=\left[\frac{(1-\phi) l}{1 / c(l)-1}\right]^{\sigma}>0, \quad \frac{\partial^{2} \chi}{\partial \phi \partial \xi}<0, \\
& \frac{\partial^{2} \chi}{\partial l \partial \xi}=\frac{\partial \chi}{\partial \xi} \frac{\partial}{\partial l} \log \frac{\partial \chi}{\partial \xi}=\sigma \frac{\partial \chi}{\partial \xi}\left[\frac{c^{\prime}}{c}+\frac{c^{\prime}}{1-c}+\frac{1}{l}\right]=\frac{\sigma}{l} \frac{\partial \chi}{\partial \xi}\left[\frac{c^{\prime} l}{(1-c) c}+1\right] \\
& =\frac{\sigma}{l} \frac{\partial \chi}{\partial \xi}\left[1-\frac{\rho}{\rho+\left[1-\varepsilon^{(1-\gamma) \sigma}\right] \lambda l_{j}}\right]=\underbrace{\frac{\sigma}{l} \frac{\partial \chi}{\partial \xi}}_{+} \underbrace{\frac{\overbrace{\left[1-\varepsilon^{(1-\gamma) \sigma}\right] \lambda l_{j}}^{\rho+\left[1-\varepsilon^{(1-\gamma) \sigma}\right] \lambda l_{j}}}{\rho+[1}<0 .}_{+}
\end{aligned}
$$

\section{References:}

Addison, J.T. and Wagner, J. (1994). "UK Unionism and Innovative Activity: Some Cautionary Remarks on the Basis of a Simple Cross-economy Test." British Journal of Industrial relations 32: 85-98.

Agell, J. and Lommerud, K.J. (1997). "Minimum Wages and the Incentives for Skill Formation." Journal of Public Economics 64: 25-40.

Aghion, P. and Howitt, P. (1998). Endogenous Growth Theory. Cambridge (Mass.): MIT Press.

Beitnes, A. and Søraas, L.H. (2003). Sources of Total Factor Productivity Growth in South Korea 1970-2000. Norwegian University of Science and Technology. Master Thesis. Department of Industrial Economics and Technology Management. Trondheim, Norway.

Belot, M. and van Ours, J.C. (2001). "Unemployment and Labor Market Institutions: An Empirical Analysis." Journal of the Japanese and International Economics 15: 403-418.

Betts, J.R., Odgers, C.W. and Wilson M.K. (2001). "The Effects of Unions on Research and Development: an Empirical Analysis using Multi-year Data." Canadian Journal of Economics 34: 785-806. 
Bronars, S.G., Deere, D.R. and Tracy, J.S. (1994). "The Effect of Unions on Firm Behavior: an Empirical Analysis using Firm-level Data." Industrial Relations 33: 426-451.

Cahuc, P. and Michel, P. (1996). "Minimum Wage Unemployment and Growth." European Economic Review 40: 1463-1482.

Connolly, R., Hirsch, B.T. and Hirschey, M. (1986). "Union Rent Seeking, Intangible Capital, and Market Value of the Firm." Review of Economics and Statistics 68: 567-577.

Dinopoulos, E. and Zhao, L. (2003). Globalization, Unionization and Efficiency Wages. Mimeo. Downloadable at:

http://bear.cba.ufl.edu/dinopoulos/research.html

Dixit, A. and Pindyck, K. (1994). Investment under Uncertainty. Princeton: Princeton University Press.

Hirsch, B.T. (1990). "Innovative Activity, Productivity Growth, and Firm Performance: are Labor Unions a Spur or a Deterrent?" Advances in Applied Micro-Economics 5: 69-104.

Hirsch, B.T. (1992). "Firm Investment Behavior and Collective Bargaining Strategy." Industrial Relations 31: 95-221.

Lingens, J. (2003). "The Impact of a Unionized Labour Market in a Schumpeterian growth Model." Labour Economics 10: 91-104.

Meckl, J. (2004). "Accumulation of Technological Knowledge, Wage Differentials, and Unemployment." Journal of Macroeconomics 26: 65-82.

Menezes-Filho, N., Ulph, D. and Van Reenen, J. (1998). "R\&D and Unionism: Comparative Evidence from British Companies and Establishments." Industrial and Labor Relations Review 52: 45-63.

Palokangas, T. (1996). "Endogenous Growth and Collective Bargaining." Journal of Economic Dynamics and Control 20: 925-944.

Palokangas, T. (2000). Labour Unions, Public Policy and Economic Growth. Cambridge (U.K.): Cambridge University Press.

Palokangas, T. (2005). "International Labour Union Policy and Growth with Creative Destruction." Review of International Economics 13: 90-105.

Peretto, P.F. (1998). "Market Power, Growth and Unemployment." Duke Economics Working Paper 98-16.

Romer, P.M. (1990). "Endogenous Technological Change." Journal of Political Economy 98: S71-S102.

Wälde, K. (1999). "A Model of Creative Destruction with Undiversifiable Risk and Optimizing Households." The Economic Journal 109: C156-C171. 